\title{
Binocular rivalry and reciprocal inhibition
}

ROBERT FOX ${ }^{2}$ AND FRANK RASCHE

VANDERBILT UNIVERSITY

Any explanation of binocular rivalry based on a reciprocal inhibition mechanism would require that unilateral increases in the stimulus strength of the rivalry target in one eye produce increases in the mean nonsuppression duration of that eye and concommitant decreases in the mean nonsuppression duration of the contralateral eye. To test that hypothesis, the stimulus strength (in this case, contrast) of one rivalry target was varied $(0.1,1,10$, and $100 \mathrm{ft}-\mathrm{L})$ while the strength of the target in the other eye remained constant. The data, obtained from six experienced Ss, indicate that variations in stimulus strength do not alter the mean nonsuppression duration of the recipient eye. This outcome offers a fundamental difficulty for the reciprocal inhibition concept. A model that assumes partially independent suppression and dominance mechanisms is suggested as a more adequate alternative.

When different stimuli strike corresponding parts of each eye, the eyes undergo a reciprocal transitory alternation in phenomenal dominance so that, at any moment in time, only the view from one eye is seen while the view of the other eye is phenomenally suppressed. This shift in phenomenal dominance, called binocular rivalry, is well-known and is easy to demonstrate. It undoubtedly represents a fundamental property of the human binocular visual system.

Binocular rivalry shares many parallels with the concept of reciprocal inhibition developed by Sherrington in his analysis of spinal reflexes. He demonstrated that flexion and extension of hind limbs are reciprocally coupled so that stimulation of one limb causes flexion of that limb and extension of the contralateral limb. When both hind limbs are stimulated simultaneously the pattern of flexion and extension occurs in an alternating fashion, the so-called "stepping reflex." Sherrington himself noted the parallel between rivalry alternation and spinal reflexes (Sherrington, 1906). Woodworth (1938) drew the same analogy. More recently, Ratliff (1962) and Crovitz and Lockhead (1967) have suggested that a similar kind of inhibitory process operates during binocular interaction. Although an explicit model of binocular rivalry based upon reciprocal inhibition has not been formally presented, it is our general impression that many people implicitly hold such a theory

Such a theory seems quite reasonable for at least two reasons. First, it is not difficult to imagine an inhibitory reciprocal network located at the point where the information from two eyes converges. Second, much of the behavioral data on rivalry is consistent with such a model.

Any version of a reciprocal inhibition model would involve the central assumption that inputs from each eye compete for a common channel in such a way that whenever the strength of one input exceeds its competitor the stronger input inhibits the weaker and thus gains access to the common channel. This requires that a single process simultaneously accomplish suppression in one eye and concommitant dominance of the other eye. Even though a detailed model of the neuronal network required for this reciprocal interaction has not been offered, the assumption is certainly not incompatible with available physiological evidence. The operation and importance of lateral inhibition in many afferent neural nets has been demonstrated (e.g., Ratliff, 1965); further, it is now known that the majority of cells in the visual cortex are controlled by either eye independently (Hubel \& Wiesel, 1962).
With respect to behavioral data, many experiments (e.g., Breese, 1899) have revealed a positive relationship between stimulus strength and the relative dominance of the recipient eye, a relationship strongly implied by a reciprocal inhibition model. That is, increases in the stimulus strength in one eye increase the predominance of that eye when predominance is defined as the percentage of time the eye is dominant. Moreover, it seems intuitively consistent with the model that rate of alternation should be a positive function of stimulus strength, and many experiments suggest that such a relationship does obtain. The fact that rivalry occurs even when the stimulus strengths in both eyes are physically equal (the case illustrated in Fig. 1a) does not pose a fundamental problem for the model, because the equal strength condition is basically an unstable state, and any number of variables, such as a nonconjugate eye movement, could change the effective strength of one of the inputs, thereby unbalancing the system and producing alternations. Thus, both increases in predominance as a function of stimulus strength and increases in rate as a function of stimulus strength are outcomes quite compatible with a reciprocal inhibition model.

But there is a further relationship between stimulus strength and the pattern of rivalry alternations that must hold if a reciprocal inhibition model is to provide an adequate account of the rivalry process. This relationship or prediction is that increases in stimulus strength in one eye should increase the average nonsuppression or dominance duration of that eye and concommitantly should increase the average suppression time of the eye receiving the weaker stimulus. That is true because the reciprocal inhibition concept assumes that suppression in one eye and nonsuppression in the other eye both result from a single process. In Fig. $1 \mathrm{~b}$ the predicted increase in suppression duration and nonsuppression duration for an average phase is illustrated for the case where the left eye stimulus strength is increased. Note carefully the distinction between the predominance of an eye defined in terms of percentage of viewing time over an observation period and the duration of each phase of rivalry alternation within the observation period. In Fig. $1 \mathrm{c}$ an increase in the strength of the left eye does result in an increase in predominance for that eye, yet there is no change in the duration of the nonsuppression period for the left eye, nor is there a change in the intervening suppression period for the right eye.

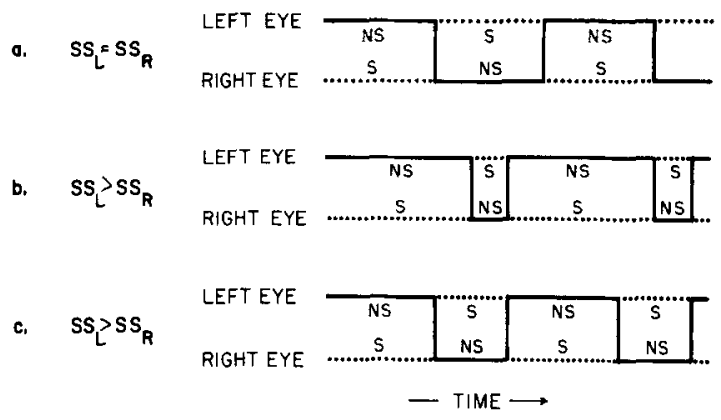

Fig. 1. Hypothesized relation between stimulus strength (SS) and duration of rivalry phase. $S$ and NS designate suppression and nonsuppression, respectively. Diagrams $b$ and $c$ represent theoretical alternatives in the case when the eyes are unequally stimulated. 
It should be clear that the pattern of rivalry alternation illustrated in Fig. Ib is the pattern predicted by the reciprocal inhibition model. On the other hand, the pattern illustrated in Fig. 1c provides an increase in predominance and in rate of alternation, but does not yield the reciprocity between suppression and nonsuppression required by the reciprocal inhibition concept.

It is difficult to determine which of these patterns is more nearly correct from the published data on rivalry alternations, because typically only summaries of statistics on predominance and rate have been reported. But there is at least one experiment that offers relevant data. Levelt (1965), working within a theoretical framework quite different from reciprocal inhibition, developed a binocular interaction model which related predominance, mean nonsuppression phase duration, and rate of alternation in rivalry. To test one of his deductions he manipulated stimulus strength (contrast) in one eye over two levels and observed the effect of the strength change upon the nonsuppression durations of the eye receiving the change of stimulus strength. He found that the nonsuppression phase durations of that eye did not change; that is, the pattern illustrated in Fig. 1c was obtained.

Motivated by the implications of these data for a reciprocal inhibition model, the present experiment investigated the effect of stimulus strength increases in one eye upon the pattern of rivalry alternation in order to secure a more definitive answer.

\section{Subjects}

\section{METHOD}

Six male Os experienced in reporting rivalry served as paid volunteers. All had normal vision.

\section{Apparatus}

The basic apparatus was an orthoptic haploscope (Wortring Troposcope) that exposed 6-deg square eye fields, transilluminated by incandescent sources and seen at optical infinity. At the eyepieces were located 2-mm artificial pupils. A head-and-chin rest minimized head movements. The rivalry targets were made from high-contrast negatives (Kodalith). The density of the dark portions was increased by opaque paint. The negatives were mounted on diffusing Plexiglas plates placed at the optical infinity positions of the troposcope. The right eye field target consisted of a $40-\mathrm{min}$ black, foveally located solid circle and a surrounding concentric 3-deg 14-min white annulus. The left eye field target consisted of a 40-min white, foveally located solid circle, a surrounding concentric 2-deg 48-min black annulus, and a concentric 3-deg 14-min white ring. The most peripheral contours of the two targets served as fusion locks while the 40-min solid circles engaged in rivalry.

Luminances were controlled by means of Wratten neutral density filters. The initial luminance of the white area of the targets without the filters was adjusted to $100 \mathrm{ft}-\mathrm{L}$. The luminance of the dark area was less than $.001 \mathrm{ft}-\mathrm{L}$. With the neutral density filters mounted behind the targets, the luminance was modified to $0.1,1.0$, and $10 \mathrm{ft}-\mathrm{L}$. A Spectra Pritchard photometer was used to calibrate the filters and periodically check the luminance levels.

The Ss reported the phenomenal changes in dominance of the center circles by means of a switch connected to one channel of an Esterline-Angus event recorder fitted with an auxiliary high-speed drive. A Hunter interval timer drove an adjacent channel to mark the 60 -sec trial period. Time resolution on the resulting charts was on the order of $50 \mathrm{msec}$ per millimeter. The data for analysis was the mean nonsuppression duration of each trial.

\section{Design and Procedure}

The luminance value of the target in one eye (constant- luminance stimulus) was held constant over a set of trials. The luminance value of the target in the other eye (variedluminance stimulus) was varied between trials. The rivalry induced by each combination of constant-luminance and varied-luminance stimuli was measured for four $60-\mathrm{sec}$ observation periods (trials) for each $S$. The set of varied-luminance values $(0.1,1,10$, and $100 \mathrm{ft}-\mathrm{L})$ was combined with two values $(10$ and $100 \mathrm{ft}-\mathrm{L})$ of the constant-luminance stimulus. Thus for each $\mathrm{S}$ a total of 32 trials was obtained (4 varied-luminance values by 2 constant-luminance values by 4 repeated trials).

The 32 trials were obtained in two $1 \frac{1 / 2-h}{h}$ sessions. Within each session the two values of the constant-luminance stimulus were paired twice with each of the variable-luminance stimuli. Order of presentation of the constant-luminance stimuli was counterbalanced between sessions. Order of presentation of the varied-luminance stimuli was counterbalanced within each session. The varied-luminance stimuli were viewed with the right eye by three of the Ss, while the other three Ss viewed those stimuli with the left eye.

The actual duration of each trial was $70 \mathrm{sec}$; the first $10 \mathrm{sec}$ were used as a warm-up and the remaining $60 \mathrm{sec}$ were recorded. The rest periods between trials ranged from 1 to 6 min with a median of $4 \mathrm{~min}$; the length of the rest period depended on the amount of rest requested by each $S$. Prior to the two experimental sessions all $\mathrm{Ss}$ received at least $1 \mathrm{~h}$ of practice in observing rivalry under conditions almost indentical with those employed in the two experimental sessions. During this practice session, and during the experimental sessions, considerable attention was paid to securing comfortable alignment of the stimulus fields so that fusion was readily maintained without strain or transitory diplopia. The Ss were instructed to report only complete changes of rivalry state. This presented no difficulty because the rivalry between the 40-min center circles was essentially unitary, with only a negligible proportion of intermediate states.

\section{RESULTS}

The statistics of interest are the significant Conditions of Luminance (constant or changing stimulation) by Levels of Varied Luminance interaction $(F=12.61$, df $=3 / 15, p<.001)$ and the trend analysis $(F=35.93, \mathrm{df}=1 / 15, \mathrm{p}<.001)$, which indicates that $95 \%$ of this interaction variance can be attributed to the different slopes of the linear components of the two conditions of luminance. Also of interest is the insignificance $(F<1)$ of the Conditions of Luminance by Levels of Varied Luminance by Levels of Constant Luminance interaction, which indicates that the two-way interaction is unaffected by the Level of Constant Luminance. The other interactions and main effects were not significant or were not relevant to this analysis.

Although the means of the nonsuppression durations for the eye receiving the varied luminance do not differ significantly, the means tend to increase for the two highest luminance levels. Inspection of the data from individual Ss reveals no systematic basis for the trend. The Newman-Keuls multiple comparison procedure indicates that the means of nonsuppression durations for the eye receiving the varied stimulation do not differ over the range of stimulation tested. However, the means for the eye receiving the constant level of stimulation differ significantly $(p=.01)$ between the levels of 0.1 and $100 \mathrm{ft}-\mathrm{L}$.

In Fig. 2 the means for the varied luminance and the constant luminance pairs are presented, with the means averaged over Ss and over the levels of constant luminance. The solid line represents the mean nonsuppression duration of the eye receiving the constant luminance stimuli, while the dotted line represents the mean nonsuppression duration of the eye receiving the varied luminance stimuli. 


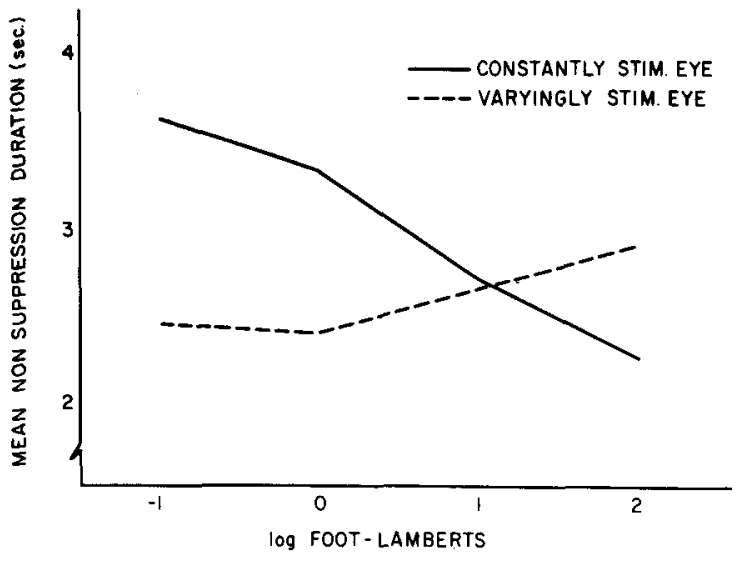

Fig. 2. Nonsuppression duration as a function of stimulus strength (contrast) in one eye.

\section{DISCUSSION}

It seems clear that the invariance of the nonsuppression phase durations, despite increases in the strength of the stimulus, is a pattern of rivalry alternation consistent with that illustrated in Fig. Ic and not consistent with the pattern illustrated in Fig. 1b. The necessary conclusion is that the reciprocal inhibition model cannot account for these data; consequently, that model is not an adequate vehicle for explaining the rivalry process. Of course, the conclusion must be qualified because the generality of the relationship over a larger range of stimulus strengths is not known, nor is it known if the relationship would hold for manipulations of stimulus strength other than contrast. Even more critical is the possibility that the concept of reciprocal inhibition presented here is oversimplified; perhaps some more sophisticated version of the reciprocal inhibition mechanism could account for the results. Yet at present such a model does not seem to be available.

But if the conclusion is correct, and if these results prove to be the general rule, then it is necessary to consider models of rivalry in which dominance and suppression operations are at least partially independent of one another. One model which could incorporate this independence might include the following features. Suppose each eye has a separate and independent suppression mechanism that generates inhibitory events of variable magnitude during each suppression phase. The duration of each suppression phase would be a function of the strength of the rivalry stimulus in that eye. The general effect of increases in stimulus strength would be to reduce the duration of the suppression phases because the stronger stimulus would tend to more readily overcome the inhibition generated by the suppression mechanism. The alternation of suppression and nonsuppression states between the eyes would be accomplished by a flip-flop switching device that would trigger the suppression mechanism alternately for each eye whenever a signal from the other eye emerged from suppression. The only function allotted to the switching device would be to alternately trigger the suppression mechanisms for the two eyes. The duration and the termination of this suppression phase would be a function of the magnitude of inhibition generated by the respective suppression mechanisms. The fact that increases in stimulus strength in one eye reduce the mean nonsuppression phase duration in the other eye could be readily accounted for by the more frequent activation of that eye's suppression mechanism; the increase in frequency of activation could come about simply because of the reduced suppression durations in the eye receiving the stronger stimulus. Obviously this kind of model requires further elaboration, but it does provide two separate mechanisms, namely, a selection device and an inhibition device, and these in turn reflect the separation or uncoupling of dominance and suppression functions that seem to be dictated by the results of this experiment.

\section{REFERENCES}

BREESE, B. B. On inhibition. Psychological Monograph, 1899, 3, 1-65. CROVITZ, H. F., \& LOCKHEAD, G. F. Possible monocular predictors of binocular rivalry of contours. Perception \& Psychophysics, 1967, 2, 83-85.

HUBEL, D. H., \& WIESEL, R. N. Receptive fields, binocular interactions, and functional architecture in the cat's visual cortex. Journal of Physiology, 1962, 160, 107-154.

LEVELT, W. J. M. On binocular rivalry. Institute for Perception RVO-TNO: Soesterberg, The Netherlands, 1965.

RATLIFF, F. Some interrelations among physics, physiology, and psychology in the study of vision. Psychology: A study of a science. Vol. 4. New York: McGraw-Hill, 1962.

RATLIFF, F. Mach bands: Quantitative studies on neural networks in the retina. San Francisco: Holden-Day, 1965.

SHERRINGTON, C. S. The integrative action of the nervous system. Cambridge: Cambridge University Press, 1906. (Republished-New Haven: Yale University Press, 1947.)

WOODWORTH, R. S. Experimental psychology. New York: Henry Holt, 1938.

\section{NOTES}

1. This research was supported by Public Health Service Research Grant NB-07619 to the senior author and Grant GY-3054 to Vanderbilt University.

2. Address: Department of Psychology, Vanderbilt University, Nashville, Tennessee 37203.

(Accepted for publication October 28, 1968.) 